\title{
Necessary complexity
}

\begin{abstract}
This month's research articles span the range of scales of gene-regulatory mechanisms, from a deceptively simple gene therapy vector, via synthetic gene expression circuits, to extremely intricate epigenetic switches. We encourage investigation of synthetic circuits exploring the functions of the 3D genome.
\end{abstract}

A minimalist modular abstraction has always driven molecular genetics, and it is appealing to consider how insights from simple synthetic circuits might complement current analysis of natural variation and selected gene edits to tackle the regulatory logic of genome expression in all its developmental and evolutionary detail. The simplicity of designed circuits becomes all the more appealing in the face of the apparently endless complexity of the regulation of gene expression in mammalian cells.

In a News \& Views (p 1164), Feilun Wu and Lingchong You point out that, despite many decades of molecular genetic manipulation of the best studied prokaryote, Escherichia coli, we still lack a toolkit of independent and compatible regulatory elements to control a gene circuit at the transcriptional level. Jeff Hasty and colleagues (p 1282) offer a way to invoke what Wu and You call a "subroutine" by controlling expression levels via plasmid copy number. This strategy is not limited to prokaryotes, as mammalian viruses, amplicons and episomes are conceptually similar. We are therefore enthusiastic to encourage abstraction of the regulatory logic of gene expression and its epigenetic control into the minimal essential components and their connections that are necessary and sufficient to deliver regulatory outcomes similar to those found in living eukaryotic cells.

Synthetic regulatory circuits designed de novo may thus provide clearer insights into the logic of gene regulation without the inherent complexity of evolved systems. However, repurposing even wellcharacterized elements of natural regulatory systems in a new context can lead to surprises. One of the simplest possible examples, analysis of just a few hundred base pairs of adeno-associated virus type 2 (AAV2), sufficient to vector therapeutic gene constructs into human cells, illustrates the difficulty of completely specifying a synthetic construct derived from an existing biological entity ( $p$ 1267). Although AAV2 fragments have been safely used as a vector in humans, these can be further improved here by eliminating an undesirable functional element, namely an enhancer-promoter cluster of binding sites for three hepatic transcription factors capable of dysregulating genes that promote hepatocellular carcinoma.

This issue of the journal also showcases genetic dissection of mammalian gene expression and regulatory elements using the evolutionary history of human populations (p 1202), transcriptional commitment of different neural and cancer cell types (pp 1239, 1261) and comparative methods using conservation between human and mouse (pp 1202, 1231, 1239). Terence Capellini, David Kingsley and colleagues ( $p$ 1202) compared coding mutations causing limb dysplasia in mice and humans and found positive selection in outof-Africa human populations upon recent GDF5 regulatory variants predisposing to osteoarthritis. They suggest that selection in Northern regions may have favored these variants that confer shorter distal-limb elements and concomitant elevated risk of osteoarthritis.

Schahram Akbarian and colleagues ( $p$ 1239) found that loss of the histone H3 lysine 9 methyltransferase encoded by Setdb1 in mature mouse neurons results in the collapse of a large topological domain encompassing a protocadherin gene cluster. Instead of stochastic single-cell expression, these cell adhesion genes are expressed from a series of smaller domains to high levels in a greater number of neurons that then gain different connectivity and function. Interestingly, a number of mouse deletions and human haplotypes in the region have been associated with cognitive phenotypes.

Johan van Nes, Rogier Versteeg and colleagues ( $p$ 1261) found that neuroblastomas adopt mesenchymal or adrenergic cell fates relevant to outcome and phenotypic plasticity. Indeed, isogenic cells can adopt either fate as they grow in vivo, suggesting that these commitments are determined epigenetically. The authors suggest a role for a feed-forward network of transcription factor genes and their extended enhancer regions bound by the same set of factors they encode, forming identity-reinforcing circuits. Transcription factor genes shared by the two states might provide a reprogramming mechanism to switch cancer cells epigenetically between alternative phenotypes.

A comprehensive resource article from the International Mouse Phenotyping Consortium ( $\mathrm{p}$ 1231) reports systematic analysis of the first 3,328 knockout mouse lines, enabling much new biology and many Mendelian disease models. When models to dissect genome function reach this scale, there is certainly plenty of room for simple, modular synthetic approaches to complement their natural complexity. 